\title{
Clustering of Russian Adjective-Noun Constructions Using Word Embeddings
}

\author{
Andrey Kutuzov \\ University of Oslo \\ Norway \\ andreku@ifi.uio.no
}

\author{
Elizaveta Kuzmenko \\ Higher School of Economics \\ Russia \\ eakuzmenko_2@edu.hse.ru
}

\author{
Lidia Pivovarova \\ University of Helsinki \\ Finland \\ pivovaro@cs.helsinki.fi
}

\begin{abstract}
This paper presents a method of automatic construction extraction from a large corpus of Russian. The term 'construction' here means a multi-word expression in which a variable can be replaced with another word from the same semantic class, for example, a glass of [water/juice/milk]. We deal with constructions that consist of a noun and its adjective modifier. We propose a method of grouping such constructions into semantic classes via 2-step clustering of word vectors in distributional models. We compare it with other clustering techniques and evaluate it against $A$ Russian-English Collocational Dictionary of the Human Body that contains manually annotated groups of constructions with nouns denoting human body parts.

The best performing method is used to cluster all adjective-noun bigrams in the Russian National Corpus. Results of this procedure are publicly available and can be used to build a Russian construction dictionary, accelerate theoretical studies of constructions as well as facilitate teaching Russian as a foreign language.
\end{abstract}

\section{Introduction}

Construction is a generalization of multi-word expression (MWE), where 'lexical variables are replaceable but belong to the same semantic class, e.g., sleight of [hand/mouth/mind]' (Kopotev et al., 2016). Constructions might be considered as sets of collocations, but they are more abstract units than collocations since they do not have a clear surface form and play an intermediate role between lexicon and grammar. A language can be seen as a set of constructions that are organized hierarchically. Thus, a speaker forms an utterance as a combination of preexisting patterns.

This view has been developed into Construction Grammar, the theory that sees grammar as a set of syntactic-semantic patterns, as opposed to more traditional interpretation of grammar as a set of rules (Fillmore et al., 1988).

Let us, for instance, consider English nearsynonyms strong and powerful. It is well-known that they possess different distributional preferences manifested in collocations like strong tea and powerful car (but not vice versa) ${ }^{1}$. These collocations are idiosyncratic and, frankly speaking, should be a part of the lexicon.

On the other hand, it is possible to look at these examples from the constructional point of view. In this sense, the former collocation would be a part of the construction 'strong [tea/coffee/tobacco/...]', while the latter would be a part of the construction 'powerful [car/plane/ship/...]'. Thus, collocations like strong tea can be considered to be parts of more general patterns, and all collocations that match the same pattern, i.e. belong to the same construction, can be processed in a similar way. This is the central idea of the constructional approach: language grammar consists of more or less broad patterns, rather than of general rules and vast amount of exceptions, as it was seen traditionally.

A constructional dictionary might be useful for both language learners and NLP systems that often require MWE handling as a part of semantic analysis. Manual compiling of construction lists is time-consuming and can be done only for some specific narrow tasks, while automatic construction extraction seems to be a more difficult task than collocation extraction due to the more abstract nature of constructions.

In this paper, we present a novel approach to

\footnotetext{
${ }^{1}$ See (Church et al., 1991) for more examples and discussion on how such regularities may be automatically extracted from corpus.
} 
construction extraction using word embeddings and clustering. We focus on adjective-noun constructions, in particular on a set of 63 Russian nouns denoting human body parts and their adjective modifiers. For each noun, the task is to cluster its adjectival modifiers into groups, where all members of a group are semantically similar, and each group as a whole is a realization of a certain construction ${ }^{2}$.

Our approach is based on the distributional hypothesis suggesting that word co-occurrence statistics extracted from a large corpus can represent the actual meaning of a word (Firth, 1957, p. 11). Given a training corpus, each word is represented as a dense vector (embedding); these vectors are defined in a multi-dimensional space in which semantically similar words are located close to each other. We use several embedding models trained on Russian corpora to obtain information about semantic similarity between words. Thus, our approach is fully unsupervised and does not rely on manually constructed thesauri or other semantic resources.

We compare various techniques to perform clustering and evaluate them against an established dictionary. We then apply the best performing method to cluster all adjective-noun bigrams in the Russian National Corpus and make the obtained clusters publicly available.

\section{Related Work}

Despite the popularity of the constructional approach in corpus linguistics (Gries and Stefanowitsch, 2004), there were few works aimed at automatic building of construction grammar from corpus. Borin et al. (2013) proposed a method of extracting construction candidates to be included into the Swedish Constructicon, which is developed as a part of Swedish FrameNet. Kohonen et al. (2009) proposed using the Minimum Description Length principle to extract constructional grammar from corpus. The common disadvantage of both studies is the lack of formal evaluation, which is understandable given the complex lexical-syntactic nature of constructions and the difficulty of the task.

Another line of research is to focus on one particular construction type, for example, light

\footnotetext{
${ }^{2}$ A group may consist of a single member, since a pure idiosyncratic or idiomatic bigram is considered an extreme case of construction with only one surface form.
}

verbs (Tu and Roth, 2011; Vincze et al., 2013; Chen et al., 2015) or verb-particle constructions (Baldwin and Villavicencio, 2002). This approach allows to make a clear task specification and build a test set for numerical evaluation. Our study sticks to the latter approach: we focus on the adjective-noun constructions, and, more specifically, on the nouns denoting body parts, because manually compiled gold standard exists for these data only.

To the best of our knowledge, the presented research is the first attempt on automatic construction extraction for Russian. The approach we employ was first elaborated on in (Kopotev et al., 2016). Their paper demonstrated (using several Russian examples) that the notion of construction is useful to classify automatically extracted MWEs. It also proposed an application of distributional semantics to automatic construction extraction. However, the study featured a rather simplistic clustering method and shallow evaluation, based on (rather voluntary) manual annotation.

Distributional semantics has been previously used in the MWE analysis, for example, to measure acceptability of word combinations (Vecchi et al., 2016) or to distinguish idioms from literal expressions (Peng et al., 2015); in the latter work, word embeddings were successfully applied.

Vector space models for distributional semantics have been studied and used for decades (see (Turney and Pantel, 2010) for an extensive review). But only recently, Mikolov et al. (2013) introduced the highly efficient Continuous skip-gram (SGNS) and Continuous Bag-ofWords (CBOW) algorithms for training the socalled predictive distributional models. They became a de facto standard in the NLP world in the recent years, outperforming state-of-the-art in many tasks (Baroni et al., 2014). In the present research, we use the SGNS implementation in the Gensim library (̌̌ehưřek and Sojka, 2010).

\section{Data Sources}

2 data sources were employed in the experiments:

1. A Russian-English Collocational Dictionary of the Human Body (Iordanskaja et al., $1999)^{3}$, as a gold standard for evaluating our approaches;

\footnotetext{
${ }^{3}$ http://russian. cornell. edu/body/
} 
2. Russian National Corpus ${ }^{4}$ (further RNC), to train word embedding models and as a source of quantitative information on word co-occurrences in the Russian language.

We now describe these data sources in more details.

\subsection{Gold Standard}

Our gold standard is A Russian-English Collocational Dictionary of the Human Body (Iordanskaja et al., 1999). This dictionary focuses on the Russian nouns that denote body parts ('рука' (hand), 'нога' (foot), 'голова' (head), etc.). Each dictionary entry contains, among other information, the list of words that are lexically related to the entry noun (further headword). These words or collocates are grouped into syntactic-semantic classes, containing 'adjective+noun' bigrams, like ‘лысая голова' (bald head).

For example, for the headword 'рука' (hand) the dictionary gives, among others, the following groups of collocates:

- Size and shape, aesthetics: 'длинные' (long), 'узкие' (narrow), 'пухлые' (pudgy), etc.

- Color and other visible properties: 'белые' (white), 'волосатые' (hairy), 'загорелые' (tanned), etc.

The authors do not employ the term 'construction' to define these groups; they use the notion of lexical functions rooted in the MeaningText Theory, known for its meticulous analysis of MWEs (Mel'cuk, 1995). Nevertheless, we assume that their groups can be roughly interpreted as constructions; as we are unaware of any other Russian data source suitable to evaluate our task, the groups from the dictionary were used as the gold standard in the presented experiments. Note that only 'adjective + noun' constructions were extracted from the dictionary; we leave other types of constructions for the future work. All the headwords and collocates were lemmatized and PoStagged using MyStem (Segalovich, 2003).

\subsection{Utilizing the Russian National Corpus}

The aforementioned dictionary is comparatively small; though it can be used to evaluate clustering approaches, its coverage is very limited.

\footnotetext{
${ }^{4}$ http://ruscorpora.ru/en
}

Thus, we used the full RNC corpus (209 million tokens) to extract word collocations statistics in the Russian language: first, to delete non-existing bigrams from the gold standard, and second, to compute the strength of connection between headwords and collocates. In particular, we calculated Positive Point-Wise Mutual Information (PPMI) for all pairs of headwords and collocates.

It is important to remove the bigrams not present in the RNC from the gold standard, since the dictionary contains a small amount of adjectives, which cannot naturally co-occur with the corresponding headword and thus are simply a noise (e.g. 'остроухий' (sharp-eared) cannot cooccur with 'yxo' (ear)). In total, we removed 36 adjectives.

After this filtering, the dataset contains 63 nominal headwords and 1773 adjectival collocates, clustered into groups. There is high variance among the headwords both in terms of collocates number-from 2 to 140 , and the number of groups-from 1 to 16 . We believe that the variety of the data represents the natural diversity among nouns in their ability to attach adjective modifiers. Thus, in our experiments we had to use clustering techniques able to automatically detect the number of clusters (see below).

We experimented with several distributional semantics models trained on the RNC with the Continuous Skip-Gram algorithm. The models were trained with identical hyperparameters, except for the symmetric context window size. The first model (RNC-2) was trained with the window size 2, thus capturing synonymy relations between words, and the second model (RNC-10) with the window size 10, thus more likely to capture associative relations between words rather than paradigmatic similarity (Levy and Goldberg, 2014). Our intention was to test how it influences the task of clustering collocates into constructions. For reference, we also tested our approaches on the models trained on the RNC and Russian Wikipedia shuffled together (with window 10); however, these models produced suboptimal results in our task (cf. Section 6).

As a sanity check, we evaluated the RNC models against the Russian part of the Multilingual SimLex999 dataset (Leviant and Reichart, 2015). On this dataset, our models produced the reasonable Spearman correlation values 0.42 for window size 2 and 0.36 for window size 10 . Thus, we 
consider them suitable for downstream semanticrelated tasks.

\section{Clustering Techniques}

We now briefly overview several clustering techniques used in this study.

\subsection{Affinity Propagation}

In most of our experiments we use the Affinity Propagation algorithm (Frey and Dueck, 2007). We choose Affinity Propagation because it detects the number of clusters automatically and supports assigning weights to instances providing more flexibility in utilizing various features.

In this algorithm, during the clustering process all data points are split into exemplars and instances; exemplars are data points that represent clusters (similar to centroids in other clustering techniques), instances are other data points that belong to these clusters. At the initial step, each data point constitutes its own cluster, i.e. each data point is an exemplar. At the next steps, two types of real-valued messages are exchanged between data points: 1) an instance $i$ sends to a candidate exemplar $k$ a responsibility that is a likelihood of $k$ to be an exemplar for $i$ given similarity (squared negative euclidean distance) between embeddings for $i$ and $k$ and other potential exemplars for $i ; 2)$ a candidate exemplar $k$ sends to $i$ an availability that is a likelihood of $i$ to belong to the cluster exemplified by $k$ given other potential exemplars. The particular formulas for responsibility and availability rely on each other and can be computed iteratively until convergence. During this process, the likelihood of becoming an exemplar grows for some data points, while for the others it drops below zero and thus they become instances.

One of the most important parameters of the algorithm is preference, which affects the initial probability of each data point to become an exemplar. It can be the same for each data point, or assigned individually depending on external data.

The main disadvantage of this algorithm is its computational complexity: it is quadratic, since at every step each data point sends a message to all other data points. However, in our case this drawback is not crucial, since we have to cluster only few instances for each headword (the maximum number of collocates is about 150).

\subsection{Spectral Clustering}

Since the number of clusters is different for each headword, we cannot use clustering techniques with a pre-defined number of clusters, like $k$ means and other frequently used techniques. That is why we employ a cascade approach where the first algorithm defines the optimal number of clusters and this number is used to initialize the second algorithm. The Spectral Clustering (Ng et al., 2001) was used for the second step; essentially, it performs dimensionality reduction over the initial feature space and then runs $k$-means on top of the new feature space.

\subsection{Community Detection}

For comparison, we test community detection algorithms (Fortunato, 2010) that take as an input a graph where nodes are words and edges are weighted by their pairwise similarities (in our case, cosine similarities).

The Spin glass algorithm (Reichardt and Bornholdt, 2006) is based on the idea of spin adopted from physics. Each node in a graph has a spin that can be in $q$ different states; spins tend to be aligned, i.e. neighboring spins prefer to be in the same state. However, other types of interactions in the system lead to the situation where various spin states exist at the same time within homogeneous clusters. For any given state of the system, its overall energy can be calculated using mathematical apparatus from statistical mechanics; spins are initialized randomly and then the energy is minimized by probabilistic optimization. This model uses both topology of the graph and the strength of pairwise relations. The disadvantage is that this algorithm works with connected graphs only.

The Infomap community detection algorithm (Rosvall et al., 2009) is based on a random walk model over networks and the Minimum Description Length principle. In this model, each node has a code that consists of two parts: a cluster code and a node code within the cluster. A trajectory of a random walker is described as a concatenation of codes of all nodes on the path. Each time a walker passes from one cluster to another, a new cluster code should be added, which makes the overall description longer; at the same time if a cluster is too big or not connected, the node codes are too long, which is also not optimal. The task is to assign optimal codes to the nodes, so that the overall description length of a 
random trajectory is minimal.

The algorithm works in an agglomerative fashion: first, each node is assigned to its own module. Then, the modules are randomly iterated and each module is merged with the neighboring module that resulted in maximum decrease of description length; if such a merge is impossible, the module stays as it is. This procedure is repeated until the state where no module can be used. Weights on the edges linking to a particular node may increase or decrease the probability of a walker to end up at this node.

\section{Proposed Methods}

The input of a clustering algorithm consists of nominal headwords accompanied with several adjectival collocates (one headword, obviously, corresponds to several collocates). For each headword, the task is to cluster its collocates in an unsupervised way into groups maximally similar to those in the gold standard ${ }^{5}$. The desired number of clusters is not given and should be determined by the clustering algorithm.

In this paper, we test 2 novel approaches compared with a simple baseline and with a community detection technique. These methods include:

1. Baseline: clustering collocates with the Affinity Propagation using their vectors in word embedding models as features.

2. Fine-tuning preference parameter in the Affinity Propagation by linking it to word frequencies, thus employing them as pointers to the selection of cluster centers.

3. Cascade: detecting the number of clusters with the Affinity Propagation (using collocates' embeddings as features), and then using the detected clusters number in spectral clustering of the same feature matrix.

4. Clustering collocates using community detection methods on semantic similarity graphs where collocates are nodes.

Below we describe these approaches in detail.

\footnotetext{
${ }^{5}$ It is also possible to instead use adjectives as entry words and to cluster nouns. In theory, each utterance may be understood as a set of corresponding and hierarchically organized constructions; e.g., any ADJ+NOUN phrase is a combination of two constructions: ADJ+X and X+NOUN. However, there is no gold standard to evaluate the latter task. The dictionary contains noun entries only, and many adjectives appear only in a couple of entries.
}

\subsection{Baseline}

The baseline approach uses Affinity Propagation with word embeddings as features and with default settings, as implemented in the scikit-learn library (Pedregosa et al., 2011).

In all our methods-the baseline and the approaches proposed in the next sections-the headword itself participates in the clustering, as if it was a collocate; at the final stage of outputting the clustering results, it is eliminated. In our experiments, this strategy consistently improved the performance. The possible explanation is that including the headword as a data point structures the network of collocates and makes it more 'connected'; the headword may also give a context and to some extend help to disambiguate polysemantic collocates.

\subsection{Clustering with Affinity Propagation}

We introduce two improvements over the baseline: fine-tuning of the Affinity Propagation and using it in pair with the spectral clustering.

\subsubsection{Fine-tuning Affinity Propagation}

Many clusters in the gold standard contain one highly frequent word around which the others group. It should be beneficial for the clustering algorithm to take this into account. There is the preference parameter in the Affinity Propagation, which defines the probability for each node to become an exemplar. By default, preference is the same for all instances and is equal to the median negative Euclidean distance between instances, meaning all instances (words) have initially equal chances to be selected as exemplars.

Instead, we make each word's preference proportional to its logarithmic frequency in the corpus. Thus, frequent words now have higher probability to be selected as exemplars, which also influences the produced number of clusters ${ }^{6}$.

All the other hyperparameters of the Affinity Propagation algorithm were kept default.

\subsubsection{Cascade clustering}

The clustering techniques that require a predefined number of clusters, such as spectral clustering, cannot be directly applied to our data. Thus, we employ Affinity Propagation to find out the number of clusters for a particular headword,

\footnotetext{
${ }^{6}$ We tried using corpus frequencies of full bigrams to this end; it performed worse than with the collocates' frequencies, though still better than the baseline.
} 


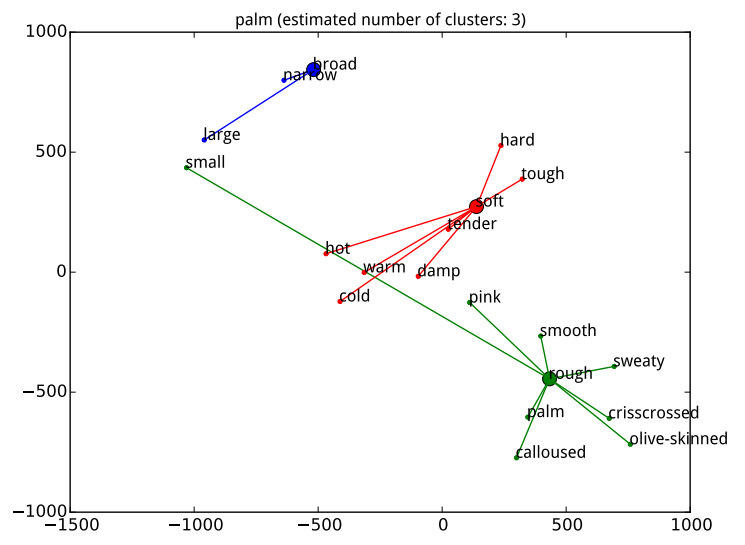

Figure 1: Clustering of the collocates for 'ладонь' (palm) by the Two-Step algorithm; the measure units on the axes are artificial coordinates used only for the 2-d projection of highdimensional word embeddings.

and then the clustering itself is done by the spectral clustering algorithm ${ }^{7}$ with the default hyperparameters.

We further refer to this method as Two-Step. Figure 1 shows a $t-S N E$ (Van der Maaten and Hinton, 2008) two-dimensional projection of an example clustering of the collocates for 'ладонь' (palm), with 'шершавый’ (rough), 'широкий’ (broad) and 'мягкий' (soft) chosen as exemplars (large dots on the plot). Note that the Russian data was used to obtain clustering; dictionary-based English translations serve only as labels in this and the following plot.

\subsection{Clustering with the Spin Glass Community Detection on Graphs}

For comparison with Affinity Propagation methods, we use community detection algorithms on semantic similarity graphs. First, a graph is constructed, in which the words (the headword and its collocates) are vertexes. Then, for each pair of vertexes, we calculate their cosine similarity in the current word embedding model. If it exceeds a pre-defined threshold, an edge between these two vertexes is added to the graph with the cosine similarity value as the edge weight. ${ }^{8}$

The Spin glass community detection algorithm

\footnotetext{
${ }^{7}$ In our preliminary experiments, we tried to use $K$-Means for the second step, but it performed worse than spectral clustering.

${ }^{8}$ The threshold is automatically adapted for each headword separately, based on the average cosine similarity between pairs of its collocates; thus, in more semantically 'dense' sets of collocates, the threshold is higher.
}

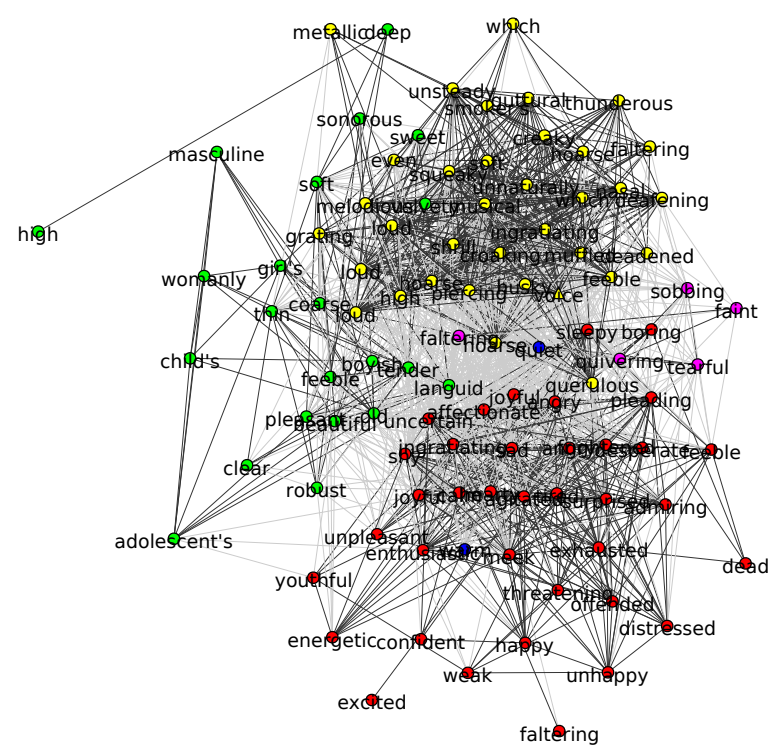

Figure 2: Clustering of the collocates for 'голос' (voice) by the Spin glass algorithm.

was employed to find clusters in the graph. Spin glass cannot process unconnected graphs; thus, if this is the case (about 10-15\% of the headwords in the gold standard), we fall back to the Infomap community detection algorithm; with connected graphs, it performs worse than Spin glass. We use the implementations of the community detection algorithms in the Igraph library (Csardi and Nepusz, 2006), and the whole gold standard as a development set to fine-tune the hyperparameters of the algorithms. Figure 2 shows the results of graph clustering for 'голос' (voice) headword, with different clusters shown in colors and edge widths representing cosine similarities. The visualization shows that the similarities between words belonging to one cluster are on average higher than those on the inter-cluster edges.

\section{Results}

We report our clustering performance as macroaverage Adjusted Rand Index (Hubert and Arabie, 1985) between the clusterings produced by our algorithms and the gold standard. The Adjusted Rand Index (ARI) is the ratio of correctly classified pairs to all pairs, adjusted for chance. All possible pairs of data points are used to compute ARI; each pair in the gold set may fall either in the same cluster or in two different clusters and the pair is counted as correctly classified if it does the same in the automatically obtained clustering. ARI values range from -1 to 1 , where 1 means 
Table 1: Clustering evaluation, average ARI and standard deviation

\begin{tabular}{l|cccc}
\hline Method & $R N C-2$ & $R N C-10$ & $R N C W-2$ & $R N C W-10$ \\
\hline Baseline & 0.22 & 0.17 & 0.17 & 0.16 \\
StDev & 0.27 & 0.23 & 0.24 & 0.24 \\
\hline Spin glass & 0.22 & 0.22 & 0.18 & 0.18 \\
StDev & 0.28 & 0.30 & 0.27 & 0.28 \\
\hline AffProp & 0.33 & 0.31 & 0.30 & 0.28 \\
StDev & 0.38 & 0.37 & 0.38 & 0.37 \\
\hline Two-Step & $\mathbf{0 . 3 4}$ & 0.33 & 0.31 & 0.29 \\
StDev & 0.36 & 0.37 & 0.37 & 0.37 \\
\hline
\end{tabular}

perfect correspondence between the gold standard and the clustering; -1 means negative correlation; 0 means the clustering and the gold standard are not related to each other.

We compute ARI individually for each headword and then average over all 63 entries. The Table 1 presents the evaluations results. RNC-2 and RNC-10 stand for the word embedding models trained on the RNC with symmetric window 2 and 10 respectively; RNCW stands for the respective models trained on the RNC and the Russian Wikipedia together. Spin glass is the method using communities detection on graphs (Section 5.3), AffProp is the single-step Affinity Propagation clustering (Section 5.2), and Two-Step is our proposed approach of cascade clustering. We also report the standard deviation of the individual headwords ARI for each approach (StDev).

As can be seen from the table, the baseline, which is a simple clustering of word embeddings, is difficult to beat. The graph-based community detection algorithm performs on par with the baseline on the models with window size 2 and only slightly outperforms it on the models with window 10. However, using the fine-tuned Affinity Propagation makes a huge difference, pushing ARI higher by at least 10 decimal points for all models. Feeding the number of clusters detected by the Affinity Propagation into the spectral clustering algorithm (our Two-Step approach) consistently increases the performance by one point more. Note that the Two-Step method is also considerably faster than the graph-based Spin glass algorithm.

It is worth noticing that the larger window mod- els consistently perform worse in this task. It seems that the reason is exactly that they pay more attention to broad associative relatedness between words and less to direct functional or paradigmatic similarity. But this is precisely what is important in the task of clustering collocates: we are trying to find groups of adjectives which can roughly substitute each other in modifying the headword noun. For example, 'beautiful' and 'charming' are equally suitable to characterize a pretty face, but 'beloved face' does not belong to the same construction; however, in the models with larger window size 'beautiful' and 'beloved' are very close and will fall into the same cluster.

At the same time, the variance among headwords may be higher than the variance between models. For example, in our experiments, for the headword 'ступня' (foot/sole), all four methods-two-step and spin glass on the RNC2 and the RNC10-yield ARI 0.816 and produce identical results. At the same time, for the headword 'живот' (stomach/belly) all four methods produced negative ARI, which probably means that clustering for this headword is especially difficult to predict.

In Figure 3 we present individual headwords ARI for the 4 best performing methods. The headwords in the plot are sorted by the number of collocates. The headwords with less than 10 collocates are excluded from the plot: these smaller entries are more diverse and in many cases yield $A R I=0$ or $A R I=1^{9}$. It can be seen from the figure that for many headwords ARI from different methods are almost identical and there are clear 'easy' and 'difficult' headwords. The more collocates the headword has the closer are the results produced by different approaches. Similar variability among headwords was observed before in various MWErelated tasks (Pivovarova et al., 2018); we assume that this can be at least partially explained by different abilities of words to form stable MWEs. Nevertheless, it can be seen from Figure 3 that in most cases ARI is higher than zero, pointing at significant correlation between the gold standard and the automatic clustering.

Another interesting finding is that the models trained on the RNC and Wikipedia together show worse results than the models trained on the RNC only, as can be seen from Table 1. Thus, despite

\footnotetext{
${ }^{9}$ However, all 63 headwords were used to compute the average values in Table 1.
} 


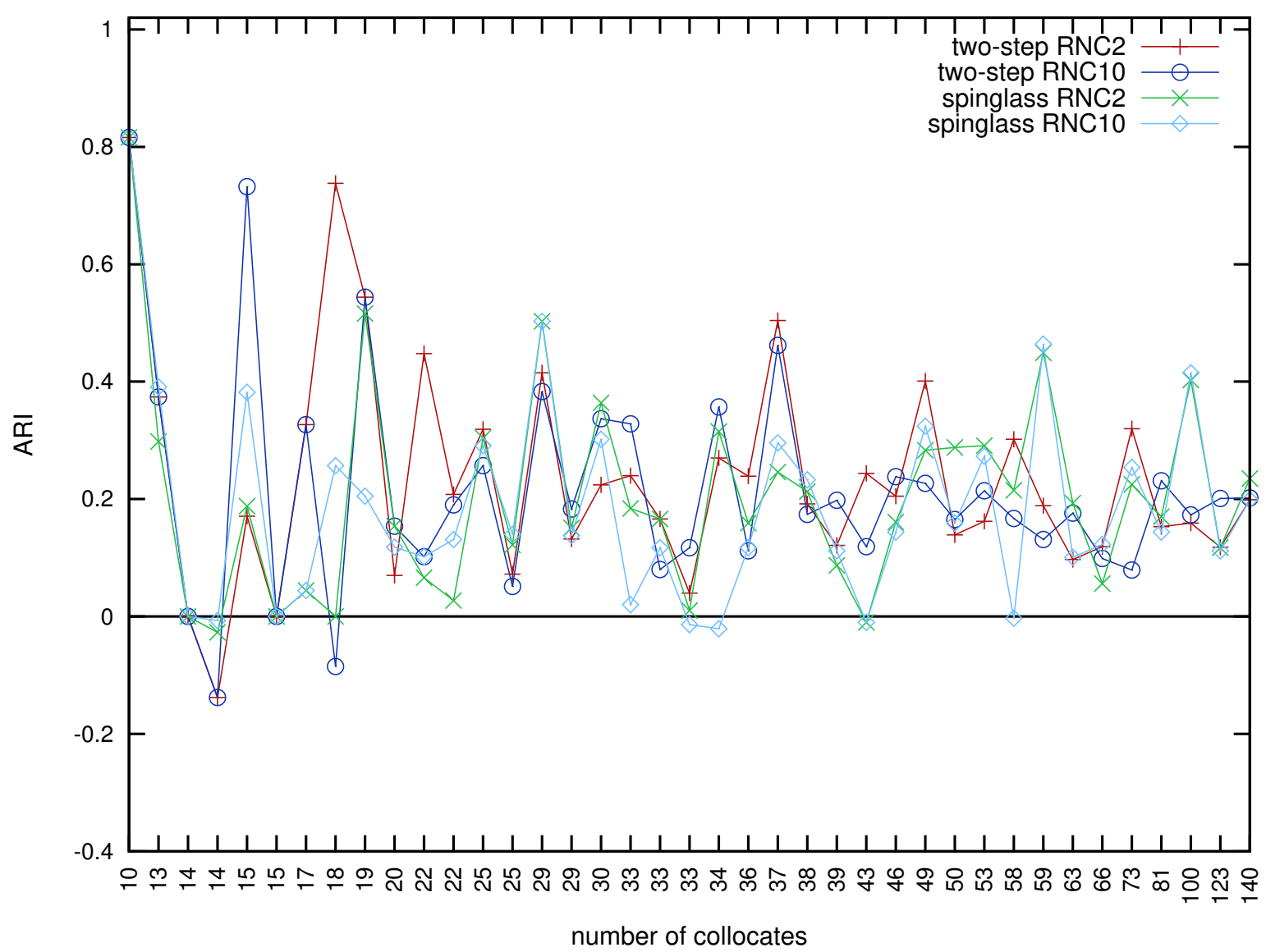

Figure 3: Individual headwords ARI for 4 best-performing methods; the headwords are sorted by the number of collocates.

the fact that the training corpus was more than two times larger, it did not result in better embeddings. This seems to support the opinion in (Kutuzov and Andreev, 2015) that when training distributional models, versatile and balanced nature of the corpus might be at least as important as its size.

Using our Two-Step algorithm and the $R N C$ 2 model, we produced clusterings for all 'adjective+noun' bigrams in the RNC with PPMI more than 1 , the corpus frequency of the bigram more than 10 and the frequency of the nominal headword more than 1000 . This corresponds to 6036 headwords and 143314 bigrams (headwords with only 1 collocate were excluded). We publish this dataset online together with our gold standard on the home page of the CoCoCo project ${ }^{10}$. For better cross-linguistic comparability, all PoS tags in these datasets were converted to the Universal PoS Tags standard (Petrov et al., 2012).

This clustering was evaluated against our gold

\footnotetext{
${ }^{10}$ Collocations, http://cosyco.ru/cococo/

Colligations,

Corpora 10
}

standard (A Russian-English Collocational Dictionary of the Human Body) as well. We had to work only with the intersection of the gold standard data and the resulting clustering, thus only a part of the gold standard was actually used for the evaluation (59 headwords out of 63, and 966 collocations out of 1758). It produced ARI=0.38 calculated on all headwords and $\mathbf{A R I}=\mathbf{0 . 3 1}$ after we excluded 6 headwords that have only one collocate in this dataset-their evaluation always produces $A R I=1$, independent of what the clustering algorithm outputs. These results confirm that the proposed algorithm performs well not only on the limited artificial data from the gold standard, but on the real world data.

Note that this is partial evaluation and many bigrams are left unattended. For example, for the headword 'лицо' (face), the collocates 'увядший' (withered) and 'морщинистый' (wrinkled) are grouped together by the algorithm, which is correct according to the gold standard, and these two collocates are used in the evalua- 
tion to compute ARI. However, in the complete clustering results these collocates are also grouped together with some other words not present in the gold standard: 'сморщенный' (withered) and 'иссохший' (exsiccated), which is probably correct, and 'отсутствующий' (absent), which is obviously wrong. As the dictionary lacks these collocates, they cannot affect the evaluation results, whether they are correct or incorrect. After analyzing the data, we can suggest that the clustering quality of the complete RNC data is more or less the same as it was for the dictionary data, but more precise evaluation would require a manual linguistic analysis.

\section{Conclusion}

The main contributions of this paper are the following:

1. We investigated MWE analysis techniques beyond collocation extraction and proposed a new approach to automatic construction extraction;

2. Several word embedding models and various clustering techniques were compared to obtain MWE clustering similar to manual grouping with the highest ARI value being 0.34 ;

3. We combined two clustering algorithms, namely the Affinity Propagation and the Spectral Clustering, to obtain results higher than can be achieved by each of this methods separately;

4. The best algorithm was then applied to cluster all frequent 'adjective+noun' bigrams in the Russian National Corpus. The obtained clusterings are publicly available and could be used as a starting point for constructional studies and building construction dictionaries, or utilized in various NLP tasks.

The main inference from our experiments is that the task of clustering Russian bigrams into constructions is a difficult one. Partially it can be explained by the limited coverage of the gold standard, but the main reason is that bigrams are grouped in non-trivial ways, that combine semantic and syntactic dimensions. Moreover, the number of clusters in the gold standard varies among headwords, and thus should be detected at the test time, adding to the complexity of the task. However, it seems that distributional semantic models can still be used to at least roughly reproduce manual grouping of collocates for particular headwords.

We believe that automatic construction extraction is a fruitful line of research that may be helpful both in practical applications and in corpus linguistics, for better understanding of constructions as lexical-semantic units.

In future we plan to explore other constructions besides 'adjective + noun'; first of all we plan to start with the 'verb+noun' constructions, since they are also present in the dictionary used as the gold standard. We would also try to find or compile other gold standards, since the dictionary we use is limited in its coverage; for example, the authors allowed only literal physical meanings of the words in the dictionary, intentionally ignoring metaphors.

In all our experiments, we used embeddings for individual words. However, it seems natural to learn embeddings for bigrams since they may have quite different semantics than individual words (Vecchi et al., 2016). It is crucial to determine bigrams that need a separate embedding and/or try to utilize already learned embeddings for individual words ${ }^{11}$.

Another interesting topic would be cluster labeling, which is finding the most typical representative of a construction, or a construction name. The Affinity Propagation outputs exemplars for each cluster, but these exemplars are not always suitable as cluster labels. For example, for the headword 'ступня' (foot) the algorithm correctly identifies the following group of adjective modifiers: ['широкий' (wide), 'узкий' (narrow), ‘большой' (large), 'маленький’ (small), 'изящный' (elegant)] with 'узкий' (narrow) being the exemplar for this class. However, in the dictionary this group is labeled 'Size and shape; aestetics', which is more suitable from the human point of view. Some kind of an automatic hypernym finding technique is necessary for this task.

Finally, we plan to use hierarchical clustering algorithms to obtain a more natural structure of high-level constructions split into smaller subgroups.

\footnotetext{
${ }^{11}$ We tried additive and multiplicative strategies (Mitchell and Lapata, 2008) to obtain bigram representations from individual word vectors, but for the present moment, they did not yield significant improvements over the baseline.
} 


\section{References}

Timothy Baldwin and Aline Villavicencio. 2002. Extracting the unextractable: A case study on verbparticles. In Proceedings of the 6th conference on Natural language learning, volume 20, pages 1-7. Association for Computational Linguistics.

Marco Baroni, Georgiana Dinu, and Germán Kruszewski. 2014. Don't count, predict! a systematic comparison of context-counting vs. context-predicting semantic vectors. In Proceedings of the 52nd Annual Meeting of the Association for Computational Linguistics (Volume 1: Long Papers), pages 238-247.

Lars Borin, Linnéa Bäckström, Markus Forsberg, Benjamin Lyngfelt, Julia Prentice, and Emma Sköldberg. 2013. Automatic identification of construction candidates for a Swedish Constructicon. In Proceedings of the workshop on lexical semantic resources for NLP at NODALIDA 2013, number 088, pages 2-11. Linköping University Electronic Press.

Wei-Te Chen, Claire Bonial, and Martha Palmer. 2015. English light verb construction identification using lexical knowledge. In $A A A I$, pages 2368-2374.

Kenneth Church, William Gale, Patrick Hanks, and Donald Kindle. 1991. Using statistics in lexical analysis. Lexical acquisition: exploiting on-line resources to build a lexicon, page 115.

Gabor Csardi and Tamas Nepusz. 2006. The Igraph software package for complex network research. InterJournal, Complex Systems, 1695(5):1-9.

Charles J. Fillmore, Paul Kay, and Mary Catherine O'Connor. 1988. Regularity and idiomaticity in grammatical constructions: The case of let alone. Language, pages 501-538.

John R. Firth. 1957. A synopsis of linguistic theory, 1930-1955. studies in linguistic analysis. Oxford: Philological Society. [Reprinted in Selected Papers of J.R. Firth 1952-1959, ed. Frank R. Palmer, 1968. London: Longman].

Santo Fortunato. 2010. Community detection in graphs. Physics reports, 486(3):75-174.

Brendan J. Frey and Delbert Dueck. 2007. Clustering by passing messages between data points. Science, 315(5814):972-976.

Stefan Th. Gries and Anatol Stefanowitsch. 2004. Extending collostructional analysis: A corpus-based perspective on alternations'. International journal of corpus linguistics, 9(1):97-129.

Lawrence Hubert and Phipps Arabie. 1985. Comparing partitions. Journal of classification, 2(1):193218.

Lidija Iordanskaja, Slava Paperno, Lesli LaRocco, Jean MacKenzie, and Richard L. Leed. 1999. A RussianEnglish Collocational Dictionary of the Human Body. Slavica Publisher.
Oskar Kohonen, Sami Virpioja, and Krista Lagus. 2009. Constructionist approaches to grammar inference. In NIPS Workshop on Grammar Induction, Representation of Language and Language Learning, Whistler, Canada.

Mikhail Kopotev, Lidia Pivovarova, and Daria Kormacheva. 2016. Constructional generalization over Russian collocations. Mémoires de la Société néophilologique de Helsinki, Collocations CrossLinguistically:121-140.

Andrey Kutuzov and Igor Andreev. 2015. Texts in, meaning out: neural language models in semantic similarity task for Russian. In Computational Linguistics and Intellectual Technologies: papers from the Annual conference "Dialogue", volume 14(21). RGGU.

Ira Leviant and Roi Reichart. 2015. Separated by an un-common language: Towards judgment language informed vector space modeling. arxiv preprint. arXiv preprint arXiv:1508.00106.

Omer Levy and Yoav Goldberg. 2014. Dependencybased word embeddings. In Proceedings of the 52nd Annual Meeting of the Association for Computational Linguistics (Volume 2: Short Papers), pages 302-308.

Igor Mel'cuk. 1995. Phrasemes in language and phraseology in linguistics. Idioms: Structural and psychological perspectives, pages 167-232.

Tomas Mikolov, Ilya Sutskever, Kai Chen, Greg S. Corrado, and Jeff Dean. 2013. Distributed representations of words and phrases and their compositionality. In Advances in Neural Information Processing Systems 26, pages 3111-3119. Curran Associates, Inc.

Jeff Mitchell and Mirella Lapata. 2008. Vector-based models of semantic composition. In Proceedings of ACL-08: HLT, pages 236-244. Association for Computational Linguistics.

Andrew Y. Ng, Michael I. Jordan, and Yair Weiss. 2001. On spectral clustering: Analysis and an algorithm. In Advances in Neural Information Processing Systems, pages 849-856. MIT Press.

F. Pedregosa, G. Varoquaux, A. Gramfort, V. Michel, B. Thirion, O. Grisel, M. Blondel, P. Prettenhofer, R. Weiss, V. Dubourg, J. Vanderplas, A. Passos, D. Cournapeau, M. Brucher, M. Perrot, and E. Duchesnay. 2011. Scikit-learn: Machine learning in Python. Journal of Machine Learning Research, 12:2825-2830.

Jing Peng, Anna Feldman, and Hamza Jazmati. 2015. Classifying idiomatic and literal expressions using vector space representations. In Proceedings of the International Conference Recent Advances in Natural Language Processing, pages 507-511. INCOMA Ltd. Shoumen, BULGARIA. 
Slav Petrov, Dipanjan Das, and Ryan McDonald. 2012. A universal part-of-speech tagset. In Proceedings of the Eighth International Conference on Language Resources and Evaluation (LREC-2012). ELRA.

Lidia Pivovarova, Daria Kormacheva, and Mikhail Kopotev. 2018. Evaluation of collocation extraction methods for the Russian language. In Quantitative Approaches to the Russian Language. Routledge.

Radim Řehůřek and Petr Sojka. 2010. Software Framework for Topic Modelling with Large Corpora. In Proceedings of the LREC 2010 Workshop on New Challenges for NLP Frameworks, pages 4550, Valletta, Malta. ELRA.

Jörg Reichardt and Stefan Bornholdt. 2006. Statistical mechanics of community detection. Physical Review E, 74(1):016110.

Martin Rosvall, Daniel Axelsson, and Carl T. Bergstrom. 2009. The map equation. The European Physical Journal Special Topics, 178(1):13-23.

Ilya Segalovich. 2003. A fast morphological algorithm with unknown word guessing induced by a dictionary for a Web search engine. In MLMTA, pages 273-280.

Yuancheng Tu and Dan Roth. 2011. Learning English light verb constructions: contextual or statistical. In Proceedings of the Workshop on Multiword Expressions: from Parsing and Generation to the Real World, pages 31-39. Association for Computational Linguistics.

Peter D. Turney and Patrick Pantel. 2010. From frequency to meaning: Vector space models of semantics. Journal of artificial intelligence research, 37(1):141-188.

Laurens Van der Maaten and Geoffrey Hinton. 2008. Visualizing data using t-SNE. Journal of Machine Learning Research, 9(2579-2605):85.

Eva M. Vecchi, Marco Marelli, Roberto Zamparelli, and Marco Baroni. 2016. Spicy adjectives and nominal donkeys: Capturing semantic deviance using compositionality in distributional spaces. Cognitive science.

Veronika Vincze, Istvan T. Nagy, and Richárd Farkas. 2013. Identifying English and Hungarian light verb constructions: A contrastive approach. In Proceedings of the 51st Annual Meeting of the Association for Computational Linguistics (Volume 2: Short Papers), pages 255-261. 